\title{
Phonembewusstheit nach schriftsprachlichem Input
}

\section{Entwicklung eines Instruments für angehende Primarschullehrkräfte}

\author{
Karin Berendes ${ }^{1} \oplus$, Wolfgang Wagner²® und Richard Böhme ${ }^{3}$ \\ 'SRH Hochschule für Gesundheit, University of Applied Health Sciences, Campus Stuttgart \\ ${ }^{2}$ Hector-Institut für Empirische Bildungsforschung, Universität Tübingen \\ ${ }^{3}$ Institut für Bildungswissenschaft, Fakultät für Humanwissenschaften, Universität Regensburg
}

\begin{abstract}
Zusammenfassung: Es wird ein standardisiertes Testverfahren zur Erfassung der Phonembewusstheit nach schriftsprachlichem Input von angehenden Primarschullehrkräften (kurz: TEPP) vorgestellt. Basierend auf der Bedeutung der Phonembewusstheit für den Schriftspracherwerb wurden Aufgaben zu deren Erfassung mit unterschiedlichen Aufgabenformaten (Multiple Choice, halboffenes Antwortformat) auf unterschiedlichen Operationsebenen (z. B. Segmentieren, Manipulieren) entwickelt. Diese Aufgaben wurden anhand einer Stichprobe von 271 Lehramtsstudierenden von fünf baden-württembergischen Pädagogischen Hochschulen sowie einer Hochschule in Bayern erprobt. Im Rahmen eines zweiparametrischen logistischen (2PL) Item-Response-Theorie-Modells wurden zunächst 14 Items aufgrund niedriger Diskriminationsparameter $(\alpha<0.80)$ ausgeschlossen. Für die verbleibenden 22 Items wurde ein einparametrisches Modell berechnet, das akzeptable Infit-Statistiken (Weighted Mean Square, WMNSQ, sowie $t$-Statistiken: $0.82 \leq$ WMNSQ $\leq 1.08,-1.9 \leq t \leq 1.2$ ) sowie eine hinreichende Person Separation Reliability von .80 ergab. Die psychometrischen Kennwerte der Itemauswahl sprechen dafür, dass das entwickelte Verfahren geeignet ist, um Leistungsunterschiede von angehenden Primarschullehrkräften mit hinreichender Genauigkeit abzubilden.

Schlüsselwörter: Phonembewusstheit, Primarschullehrkräfte, Testkonstruktion, Schriftspracherwerb
\end{abstract}

Phonemic Awareness after Written Input: Development of an Instrument for Prospective Elementary School Teachers

Abstract: This article introduces a standardized test to assess phonemic awareness following written input among prospective elementary school teachers. The items measuring phonological awareness at the phoneme level were constructed based on the importance of phonemic awareness for the acquisition of reading and writing. They have different item formats (multiple choice, open-ended answer format) at different levels of operation (e.g., segmentation, manipulation). Because standardized tests to assess skills in this field are still missing in the German-speaking countries, the available study aimed at closing this gap. The sample comprised 271 student teachers from five teacher-education universities in the state of Baden-Wuerttemberg and one university in Bavaria, Germany. 14 items were excluded within a two-parametric logistic (2PL) item-response theory model because of low item-discrimination parameters $(\alpha<0.80)$. For the remaining 22 items, we estimated a one-parametric model that showed acceptable infit statistics (weighted mean square, WMNSQ, and $t$-statistics: $0.82 \leq$ WMNSQ $\leq 1.08,-1.9 \leq t \leq 1.2$ ) as well as a sufficient person separation reliability of .80 . The psychometric values of the item selection indicate that the instrument developed is suitable for displaying performance differences of prospective elementary school teachers with a sufficient accuracy.

Keywords: phonemic awareness, elementary school teacher, test construction, literacy acquisition

\section{Theoretischer Hintergrund}

Schriftsprachliche Kompetenzen tragen maßgeblich zum Bildungserfolg bei und sind nahezu unverzichtbar für eine erfolgreiche Teilhabe am gesellschaftlichen Leben (Schneider, 2017). Ein erfolgreicher Start in den Schriftspracherwerb wirkt sich langfristig positiv auf spätere Leistungen aus (Sparks, Patton \& Murdoch, 2014) und frühe Defizite können später nur schwerlich wieder auf- geholt werden (Moats, 1999). Eine Schlüsselkompetenz für einen erfolgreichen Schriftspracherwerb ist, unabhängig von der untersuchten Sprache und Orthographie, phonologische Bewusstheit (für einen Überblick siehe Ziegler \& Goswami, 2005; für einen Überblick zum deutschsprachigen Raum siehe auch Pfost, 2015; Schneider, 2017). Dabei variiert die Bedeutung der phonologischen Bewusstheit im Vergleich zu anderen Prädiktoren und in Abhängigkeit von der linguistischen Ebene. So 
kommt im Deutschen, einer Sprache mit Alphabetschrift und relativ konsistenter Orthographie, insbesondere der phonologischen Bewusstheit auf Phonemebene (Phonembewusstheit) Bedeutung zu (für die theoretische Fundierung siehe z.B. Schnitzler, 2008; für die Datenbasierung siehe z.B. Metaanalyse von Pfost, 2015). Daher sollten Kompetenzen in diesem Bereich im schriftsprachlichen Anfangsunterricht vermittelt werden (Klicpera \& Gasteiger-Klicpera, 1995). Damit angehende Primarschullehrkräfte über die notwendigen Voraussetzungen verfügen, entsprechende Übungen kompetent anleiten zu können, ist der eigene Erwerb einer ausgeprägten expliziten Phonembewusstheit ein wichtiges Ziel der Lehrkräfteausbildung (Driver, Pullen, Kennedy, Williams \& Ely, 2014).

\section{Phonembewusstheit: Konstrukt- beschreibung, Bedeutung für den Schriftspracherwerb, Entwicklung und Förderung}

Konstruktbeschreibung. Phonembewusstheit ist Teil der phonologischen Bewusstheit, die ihrerseits Teil der metalinguistischen Bewusstheit (z. B. Gillon, 2004; Purvis, McNeill \& Everatt, 2016) und der phonologischen Informationsverarbeitung (Wagner \& Torgesen, 1987) ist. Phonologische Bewusstheit wird definiert als die Fähigkeit, über die phonologische Struktur eines Wortes reflektieren zu können, ohne dabei auf die Bedeutung des $\mathrm{zu}$ analysierenden sprachlichen Materials einzugehen (Tunmer \& Hoover, 1992). Gemäß des zweidimensionalen Konstrukts zur phonologischen Bewusstheit (z. B. Fricke, Stackhouse \& Wells, 2007) lassen sich Aufgaben zur phonologischen Bewusstheit zum einen hinsichtlich der ,Größe der phonologischen Einheit' (Silbe, OnsetReim, Phonem) unterscheiden (siehe dazu auch das Modell der Hierarchischen Struktur von Wörtern nach Seymour \& Evans, 1994). Der Reim ist dabei definiert als der Teil der Silbe, der sich reimen lässt (z.B. Kr-aft $\mathrm{S}$-aft). Den fakultativen Onset bilden alle davor vorkommenden Konsonanten ( $\mathrm{Kr}-\mathrm{aft}, \underline{\mathrm{S}}$-aft). Aufgaben auf Silben- sowie Onset-Reim-Ebene werden im deutschsprachigen Raum mitunter als ,phonologische Bewusstheit im weiteren Sinne" bezeichnet (z. B. Fischer \& Pfost, 2015). Onset und Reim setzen sich wiederum aus Einzellauten, den sogenannten Phonemen, zusammen. Phoneme sind die kleinsten bedeutungsunterscheidenden Einheiten unserer Sprache (z. B. $<$ Land $>$ [lant] $-<$ Rand $>$ [вant], $<$ wohnen> ['vo:nən] - <schonen> ['Jo:nən], Graefen \& LiedtkeGöbel, 2020, S. 212). Aufgaben, die ausschließlich eine Phonembewusstheit erfordern, werden im deutschsprachigen Raum auch „phonologische Bewusstheit im engeren Sinne" genannt (z. B. Pfost, 2015). Zum anderen las- sen sich Aufgaben zur phonologischen Bewusstheit hinsichtlich der ,Explizitheit der Operation' (Identifizieren, Segmentieren, Synthetisieren und Manipulieren) unterscheiden (Fricke et al., 2007). Dabei erfordert Identifizieren das Erkennen, Segmentieren das Erkennen und Untergliedern, Synthetisieren das Erkennen und Zusammenziehen und Manipulieren das Erkennen, Untergliedern, Verändern (Weglassen, Hinzufügen, Vertauschen, Austauschen) und Zusammenziehen von phonologischen Einheiten.

Bedeutung für den Schriftspracherwerb. Im Deutschen kommt insbesondere der phonologischen Bewusstheit auf Phonemebene Bedeutung für den Schriftspracherwerb zu (Metaanalyse von Pfost, 2015; zur besonderen Bedeutung der Phonemebene für das Erlernen von Alphabetschriften siehe auch Caravolas, Volin \& Hulme, 2005). In der alphabetischen Phase des Schriftspracherwerbs basiert sowohl das Schreiben als auch das Lesen auf einem sequentiellen phonologischen Rekodierungsprozess. Beim dekodierenden Lesen müssen visuelle Reize (Grapheme) erkannt und in phonologische Informationen (Phoneme) übersetzt werden. Beim lautgetreuen Schreiben müssen phonologische Informationen wahrgenommen und in graphische Zeichen überführt werden (Berendes, Schnitzler, Willmes \& Huber, 2010). Eine ausgeprägte Phonembewusstheit ermöglicht es Kindern, diesen regelhaften Zusammenhang von Graphemen und Phonemen zu begreifen und ein grundlegendes Verständnis des alphabetischen Prinzips der Schriftsprache zu erwerben (Muter, Hulme, Snowling \& Taylor, 1998; Schnitzler, 2008; Berendes \& Weinert, 2016).

Entwicklung. Die Entwicklung der phonologischen Bewusstheit kann als ein Kontinuum angesehen werden und vollzieht sich innerhalb der Größe der linguistischen Einheit von großen zu kleinen Einheiten (Silbe $\rightarrow$ OnsetReim $\rightarrow$ Phonem) und innerhalb der Explizitheit der Operation von einfachen, impliziten zu komplexen, expliziteren Operationen (Identifizieren $\rightarrow$ Segmentieren / Synthetisieren $\rightarrow$ Manipulieren) (für einen Überblick siehe Berendes \& Weinert, 2016, S. 407 ff). Der Aufgabentyp „Identifizieren von Silben“ kann somit als der einfachste und der Aufgabentyp „Manipulieren von Phonemen“ als der schwierigste der zwölf Aufgabentypen angesehen werden (ebd.). Aktuell gibt es weder einen klaren Konsensus noch ausreichend Daten zu der Frage, ob die Schwierigkeit einer Aufgabe mehr durch die Größe der linguistischen Einheit oder durch die Explizitheit der Operation determiniert wird. Belastbare vergleichende Aussagen zu der Itemschwierigkeit von zwei Aufgaben, die in beiden Klassifikationsmerkmalen variieren und bei denen eine der beiden Aufgaben eine leichter zu bearbeitende, größere linguistische Einheit betrifft, zugleich aber eine schwierigere (komplexere, explizitere) Operation er- 
fordert, sind daher derzeit nicht möglich (Berendes \& Weinert, 2016).

Erste, implizite phonologische Bewusstheit (typische Aufgaben sind z.B. Silben klatschen, Reime erkennen) erwerben Kinder schon zu Beginn des Kindergartenalters (Schaefer, Stackhouse \& Wells, 2016). Explizite phonologische Bewusstheit (typische Aufgaben sind z. B. Phonemreihen umdrehen oder einzelne Phoneme in Wörtern zählen) entwickelt sich insbesondere im ersten Jahr des schriftsprachlichen Anfangsunterrichts (Schnitzler, 2008). Etwa ab dem zweiten Schuljahr, wenn die Schülerinnen und Schüler basale Schriftsprachfähigkeiten erworben haben, werden bei der Bearbeitung von Aufgaben zur phonologischen Bewusstheit auch orthographische Wortformen mitaktiviert und es treten vermehrt sogenannte orthographische Interferenzeffekte auf (Polich, McCarthy, Wang \& Donchin, 1983). Kennzeichnend für derartige Effekte ist, dass orthographisches Wissen die Leistung bei Aufgaben zur phonologischen Bewusstheit beeinflusst. So kann es beispielsweise bei Aufgaben zur Phonemsegmentation vorkommen, dass Buchstaben statt Phoneme gezählt werden (für Befunde im deutschsprachigen Raum siehe Schulte-Körne, 2001). Solche Interferenzeffekte können bis in das Erwachsenenalter hinein bestehen bleiben (Castles, Holmes, Neath \& Kinoshita, 2003; Seidenberg \& Tanenhaus, 1979).

Förderung. Es gilt als weitgehend gesichert, dass phonologische Bewusstheit effektiv gefördert werden kann und sich eine Förderung positiv auf den Schriftspracherwerb auswirkt (Bus \& van Ijzendoorn, 1999; Ehri et al., 2001; National Institute of Child Health and Human Development [NICHD], 2000; für den deutschsprachigen Raum siehe Fischer \& Pfost, 2015). Effekte einer Förderung der phonologischen Bewusstheit fallen für den deutschsprachigen Raum jedoch geringer aus als etwa für den englischsprachigen Raum (Fischer \& Pfost, 2015). Die geringeren Effektstärken sind vermutlich darauf zurückzuführen, dass in deutschsprachigen Schulen (anders als in englischsprachigen Schulen) zumeist rein phonetische Methoden im schriftsprachlichen Anfangsunterricht angewendet werden und die in den Evaluationsstudien untersuchten Kontrollgruppen somit ebenfalls eine phonologische Förderung erhalten haben. Dadurch vermindern sich Unterschiede zwischen Trainings- und Kontrollklassen (Einsiedler, Frank, Kirschhock, Martschinke \& Treinies, 2002). Dennoch konnten speziell bei Schülerinnen und Schülern mit niedrigen Ausgangsleistungen bedeutsam bessere Leistungszuwächse im Bereich der phonologischen Bewusstheit durch eine Förderung nachgewiesen werden (ebd.; Pfost, Blatter, Artelt, Stanat \& Schneider, 2019). Es kann angenommen werden, dass eine Förderung der phonologischen Bewusstheit im Anfangsunterricht insbesondere für Kinder mit Deutsch als Zweit- sprache gewinnbringend ist, da „beim Erwerb einer zweiten (Schrift-)Sprache neben der Artikulation in der L2 auch die phonologische Bewusstheit angeeignet werden muss, mit der das zu lernende Schriftsystem beurteilt wird“ (Jeuk, 2017, S. 278; siehe auch Blatter, 2015). Auch im inklusiven Unterricht kommt einer Förderung der phonologischen Bewusstheit besondere Bedeutung $\mathrm{zu}$ (Berkemeier \& Drinhaus, 2014; Diehl, 2017; Mußmann, 2012). Daher ist die Förderung der phonologischen Bewusstheit eine wesentliche Aufgabe des schriftsprachlichen Anfangsunterrichts (Klicpera, Schabmann, Gasteiger-Klicpera \& Schmidt, 2017) und Lehrkräfte sind dazu angehalten, entsprechende Übungen in ihren Unterricht $\mathrm{zu}$ integrieren (Ministerium für Kultus, Jugend und Sport Baden-Württemberg, 2016). Hierfür existieren verschiedene Trainingsprogramme, die entweder alleinig auf eine Förderung der phonologischen Bewusstheit abzielen oder derartige Übungen mit anderen Aufgaben (z.B. Aufgaben zur Graphem-Phonem-Korrespondenz) kombinieren und deren bereichsspezifische Wirksamkeit im Schulalter belegt wurde (z. B. „Lautarium“ von Klatte, Steinbrink, Bergström \& Lachmann, 2017 und „Phonit“ von Stock \& Schneider, 2011 - für eine Zusammenfassung zur Wirksamkeit siehe Steinbrink \& Lachmann, 2014). Aber auch der Einsatz von selbstkonzipierten Einheiten zur Förderung der phonologischen Bewusstheit stellt eine gängige Unterrichtspraxis dar.

\section{Phonembewusstheit von (angehenden) Lehrkräften}

Damit solche Übungen die gewünschten positiven Effekte haben, ist es notwendig, dass sich Lehrkräfte möglicher orthographischer Interferenzeffekte bewusst sind und orthographische von phonologischen Informationen entkoppeln. So haben beispielsweise die Anlaute der Wörter $<$ Äpfel $>$ und $<$ Ente $>$ die gleiche phonologische Qualität (['epfl], ['entə]); ihre orthographische Repräsentation unterscheidet sich jedoch. Um dies zu erkennen, ist eine ausgeprägte explizite Phonembewusstheit vonnöten, die über das erforderliche Niveau für einen erfolgreichen Schriftspracherwerb hinausgeht (Moats, 1994; Pittman, Zhang, Binks-Cantrell, Hudson \& Joshi, 2020; Spencer, Schuele, Guillot \& Lee, 2008).

In diesem Zusammenhang stellt sich die Frage, ob (angehende) Lehrkräfte tatsächlich über eine ausreichende explizite Phonembewusstheit verfügen. Dieser Frage wurde vornehmlich im englischsprachigen Forschungsraum, insbesondere den USA, nachgegangen (Joshi, Washburn \& Kahn-Horwitz, 2016). Diverse Ergebnisse deuten darauf hin, dass "significant numbers of beginning special and general education teachers [...] lack skill in analy- 
zing written words into phonemes" (Cheesman, McGuire, Shankweiler \& Coyne, 2009, S. 270). Cheesman et al. (2009) fanden beispielsweise heraus, dass eine bedeutsame Anzahl an neu anfangenden Sonder- sowie Allgemeinpädagoginnen und -pädagogen nicht qualifiziert ist, um Wörter in Phoneme zu segmentieren. Auch Spencer et al. (2008) stellten fest, dass es den von ihnen untersuchten Erstklasslehrkräften sowie Sonderpädagoginnen und -pädagogen an den notwendigen phonologischen Fähigkeiten fehlte, um eine fehlerfreie Förderung der Phonembewusstheit anbieten zu können. Im Einklang damit fanden Washburn, Binks-Cantrell, Joshi, Martin-Chang und Arrow (2016), dass die von ihnen untersuchten angehenden Lehrkräfte aus England nur ein Viertel (24\%) der Aufgaben zur Phonembewusstheit korrekt lösten, bei denen Phoneme identifiziert (ein Item), segmentiert (ein Item) und manipuliert (vier Items) werden mussten. Ähnliche Ergebnisse liefert eine Untersuchung von Stark, Snow, Eadie und Goldfeld (2016) (siehe auch Aro \& Björn, 2016 für das Finnische). Darüber hinaus zeigte eine Untersuchung von Binks-Cantrell, Washburn, Joshi und Hougen (2012), dass auch Hochschullehrkräfte, die die Ausbildung angehender Lehrkräfte mitverantworten, ein defizitäres Wissen im Bereich von grundlegenden sprachlichen Konzepten wie z. B. der Phonembewusstheit besitzen.

Im deutschsprachigen Raum fehlen bislang Untersuchungen dazu, wie gut angehende Primarschullehrkräfte im Bereich der Phonembewusstheit qualifiziert sind. Dies liegt nicht zuletzt daran, dass kein entsprechendes Verfahren existiert. Es besteht somit Bedarf für ein Instrument, mit dem die Phonembewusstheit von (angehenden) Primarschullehrkräften mit Blick auf den frühen Schriftspracherwerb erfasst werden kann.

\section{Die vorliegende Untersuchung}

In der vorliegenden Studie wurde ein Instrument zur Erfassung der Phonembewusstheit von angehenden Lehrkräften entwickelt ${ }^{1}$, das in verschiedenen Forschungsund Praxiskontexten eingesetzt werden kann. Der Fokus von TEPP (Testverfahren zur Erfassung der Phonembewusstheit nach schriftsprachlichem Input von angehenden Primarschullehrkräften) liegt auf der Phonemebene, da dieser linguistischen Ebene im deutschsprachigen Raum eine große Bedeutung im Anfangsunterricht zukommt. Im Beitrag wird die Entwicklung des Instruments dargelegt. Zum einen wird die Konstruktion der Items beschrieben und zum anderen werden Ergebnisse einer
Studie, in der TEPP erstmals an 271 angehenden Lehrkräften erprobt wurde, präsentiert.

\section{Methode}

\section{Konstruktion des Testinstruments}

Da TEPP das Leistungsniveau im Bereich der Phonembewusstheit messen sollte, wurde das Instrument als Powertest konzipiert. Auf Grundlage dieser Vorgaben wurden 55 Items konstruiert.

Inhaltsvalidität. Aufgrund der besonderen Bedeutung der Phonemebene für die Einsicht in das alphabetische Prinzip des deutschen Schriftsprachsystems sind alle Items der Phonembewusstheit zuzuordnen. Alle Aufgaben wurden von vier Expertinnen und Experten mit sprachwissenschaftlicher Expertise übereinstimmend als Aufgaben zur Phonemebene eingeschätzt. Die Aufgaben erfordern bei der Bearbeitung unterschiedliche Operationen (Phoneme Identifizieren - PI, Phoneme Segmentieren - PS, Phoneme Manipulieren - PM) und damit einhergehend unterschiedliche kognitive Prozesse. Es wurden jedoch keine Aufgaben zum Synthetisieren von Phonemen für das Instrument konstruiert, da diese bei der Bearbeitung fundierte Kenntnisse einer Lautschrift, z. B. dem Internationalen Phonetischen Alphabet (IPA), bedurft hätten.

Itemformat. Der Itempool umfasste Items in geschlossenem (Multiple Choice) und halboffenem Antwortformat. Die Multiple Choice-Items enthielten je drei Distraktoren, um die Ratewahrscheinlichkeit zu minimieren. Die falschen Antwortalternativen wurden dabei unter der Prämisse von Attraktivität und Plausibilität entwickelt, sodass sie für angehende Lehrkräfte mit unzureichender Phonembewusstheit den Anschein erweckten, korrekt zu sein bzw. sie von der korrekten Antwortmöglichkeit ablenkten. Es wurde zudem auf die Anordnung der Items geachtet, um zu verhindern, dass diese das fortlaufende Antwortverhalten beeinflusst und letztlich zu Aktualisierungseffekten führt. Bei den Items im halboffenen Antwortformat war je nach erforderter Operation jeweils eine eigenständige Kurzantwort verlangt, die aus exakt einem Buchstaben, einem Wort oder einer Zahl bestand (abgewandelte Beispielitems werden im Elektronischen Supplement 1 zur Verfügung gestellt).

Da die Schwierigkeit von Aufgaben zur phonologischen Bewusstheit auch durch sogenannte Nebenfaktoren beeinflusst wird (Berendes \& Weinert, 2016; Smith, Sim-

TEPP kann gerne für den Einsatz für wissenschaftliche Zwecke zur Verfügung gestellt werden. Entsprechende Anfragen können an die Autorin und Autoren gerichtet werden. 
mons \& Kameenui, 1998; Schnitzler, 2008), wurde bei der Itemkonstruktion darauf geachtet, diese bei allen Operationen gleichermaßen $\mathrm{zu}$ variieren. Relevante Nebenfaktoren sind die Sonorität, die Intonation, die Position der phonologischen Einheit, die bearbeitet werden soll, sowie die Komplexität der phonologischen Umgebung, in die die phonologische Einheit eingebettet ist (ebd.). Des Weiteren gehen Schwierigkeiten bei der Bearbeitung von Aufgaben zur Phonembewusstheit nach schriftsprachlichem Input nicht selten auf orthographische Interferenzeffekte zurück. Um solche Effekte gezielt zu provozieren, wurden Wörter mit orthographischen und / oder phonologischen Besonderheiten (z. B. Auslautverhärtungen, Affrikaten und Diphthonge) in die Items eingebaut.

Testfairness. Die Items wurden grundsätzlich geschlechtsneutral formuliert, um das Risiko von stereotype threats $\mathrm{zu}$ minimieren (Cadinu, Maass, Rosabianca \& Kiesner, 2005). Um begriffliche und definitorische Schwierigkeiten zu vermeiden, stand allen Teilnehmenden während der gesamten Testung ein Beiblatt zur Verfügung, auf dem elementare Aspekte zur Phonembewusstheit erläutert waren (z.B. die Definition von Phonembewusstheit und die Zeichen zur Unterscheidung von Phonemen und Graphemen). Darüber hinaus wurde auf eine geringe sprachliche Komplexität der Aufgabentexte geachtet.

Pretests. Im Vorfeld der Testung wurden kognitive Interviews mit (angehenden) Lehrkräften durchgeführt, um durch das Verbalisieren der gedanklichen Prozesse beim Bearbeiten erste Hinweise auf Verständnisschwierigkeiten der Items und die Inhaltsvalidität des Instruments zu bekommen. Zudem wurde die Eignung der Distraktoren bei Multiple Choice-Aufgaben unter Verwendung eines onlinebasierten Pretests mit 44 praktizierenden Lehrkräften überprüft. Items, die sich bei dieser ersten Erprobung als problematisch erwiesen, z. B. weil sie missverständlich formuliert waren, wurden überarbeitet oder aus dem Itempool entfernt. So wurden von den 55 konstruierten Items 36 für die weiteren Analysen selektiert.

\section{Stichprobe und Untersuchungsablauf}

Stichprobe. Es wurden $N=271$ Studierende des Grundschullehramts in die Untersuchung einbezogen. Es wurden nur Studierende berücksichtigt, die zum Zeitpunkt der Untersuchung bereits Lehrveranstaltungen besucht hatten, die phonologische Bewusstheit zum Gegenstand haben. Davon wurden $n=148$ Studierende (96\% weiblich $)^{2}$ an fünf der sechs baden-württembergischen Pädagogischen Hochschulen (Heidelberg, Karlsruhe, Ludwigsburg, Schwäbisch Gmünd und Weingarten) untersucht, die sich vornehmlich im höheren Fachsemester befanden $(M=6.91 ; S D=1.29$; $\operatorname{Min}=3 ; \operatorname{Max}=9)$. In Zusammenhang mit der verpflichtenden Wahl eines Vertiefungsfachs an Pädagogischen Hochschulen in BadenWürttemberg gaben $n=133$ Studierende (90\%) Deutsch und $n=14$ Studierende (9\%) Mathematik als solches an (ein Fall von Item-Nonresponse).

Weiterhin wurden $n=123$ Studierende des Grundschullehramts (93\% weiblich) an der Universität Regensburg in die Untersuchung einbezogen, die sich ebenfalls überwiegend im höheren Fachsemester befanden $\left(M=6.61 ; S D=1.71 ; \operatorname{Min}=3 ; \operatorname{Max}=10^{3}\right)$. An bayerischen Universitäten ist Deutsch entweder als Unterrichtsfach oder als Didaktikfach zu studieren, wobei im Unterrichtsfach deutlich mehr Leistungspunkte zu erbringen sind. $n=28$ Studierende (23\%) gaben Deutsch als Unterrichtsfach und $n=95$ Studierende (77\%) Deutsch als Didaktikfach an.

Durchführung der Untersuchung. Die Daten wurden in insgesamt vierzehn Gruppenerhebungen (Gruppengröße: $6 \leq n \leq 34$ ) im Kontext der jeweiligen Seminarsitzungen durch geschulte Testleitende erhoben. Die Durchführungsobjektivität war durch eine standardisierte Durchführung (schriftlich vorgegebene Instruktion, genaue Anweisungen zur Testdurchführung) gegeben.

Auswertung der Ergebnisse. Die Auswertung der Ergebnisse erfolgte standardisiert nach dem richtig-falschPrinzip (Punktvergabe für richtige Antworten). Somit ist eine hohe Auswertungs- und Interpretationsobjektivität gegeben.

\section{Analysestrategie}

Die Skalierung erfolgte auf Basis der Item-ResponseTheorie (IRT; de Ayala, 2009; Rost, 2004). Dabei wurde ein einparametrisches logistisches Modell (1PL, jeweils in Form eines Rasch-Modells spezifiziert) angestrebt, da dort die relativen Itemschwierigkeiten entlang des Fähigkeitskontinuums als konstant zu betrachten sind. Dadurch wird die Interpretation der Ergebnisse erleichtert, da sich (tendenziell) leichte und (tendenziell) schwierige Items voneinander abgrenzen und verschiedenen inhaltlich interpretierbaren Kompetenzniveaus zuordnen las-

\footnotetext{
Diese Zahlen sind repräsentativ für die Geschlechterverteilung im Grundschullehramtsstudium in Baden-Württemberg (Statistisches Landesamt Baden-Württemberg, 2018).

3 Die Regelstudienzeit für das Grundschullehramt beträgt in Baden-Württemberg acht und in Bayern sieben Semester.
} 
sen. Da im einparametrischen Modell identische Itemdiskriminationen angenommen werden, wurden zunächst die Diskriminationsparameter $\alpha_{i}$ mithilfe des zweiparametrischen logistischen (2PL-) Modells unter Verwendung von ConQuest ( $\mathrm{Wu}$, Adams, Wilson \& Haldane, 2007) berechnet und deren (approximative) Homogenität anhand eines Modellfitvergleichs mit einem 1PL-Modell anhand der Informationskriterien AIC (Akaike's Information Criterion; Akaike, 1974) und BIC (Bayesian Information Criterion; Schwarz, 1978) überprüft (siehe dazu auch Pohl \& Carstensen, 2012). Die Diskriminationsparameter $\alpha_{i}$ liegen nach de Ayala (2009) idealiter bei $\geq 0.80$. Items mit geringerer Diskrimination wurden entsprechend in der vorliegenden Studie ausgeschlossen, um die Itemhomogenität $\mathrm{zu}$ erhöhen sowie das Verhältnis von Testdauer zur Reliabilität der Testscores zu optimieren. Anschließend wurden die Itemfit-Statistiken (Weighted Mean Square oder Infit-Kriterium, WMNSQ) der verbleibenden Items im Rahmen eines einparametrischen (1PL-) Modells überprüft. Zusätzlich wurden basierend auf theoretischen Überlegungen mehrdimensionale 1PL-Modelle getestet (ConQuest): Ein dreidimensionales Modell mit (korrelierten) latenten Variablen für Items bezogen auf die Bereiche PI, PS und PM, sowie ein sogenanntes Testlet-Modell (Wang \& Wilson, 2005) mit einem Generalfaktor und spezifischen Faktoren für die drei Bereiche, wobei sämtliche Faktoren als miteinander unkorreliert angenommen werden. Ergänzend zu den theoriebasierten mehrdimensionalen Modellen wurde die Dimensionalität anhand exploratorischer Faktorenanalysen für kategoriale Daten untersucht (Maximum Likelihood-Schätzung mit orthogonaler Geomin-Rotation in Mplus, Version 8.4; Muthén \& Muthén, 1998-2017). Auf Basis des 1PL-Modells wurden Weighted Likelihood Estimates (WLEs; Warm, 1989) Punktschätzer für die Personenfähigkeit generiert und als Reliabilitätsmaß die WLE Person Separation Reliability (WLE PSR; Andrich, 1982) bestimmt. Die WLE PSR entspricht dem Varianzanteil der WLEs, der nicht auf Messfehler (durchschnittlicher quadrierter Standardfehler der WLEs) zurückgeht. Um die Passung der Itemschwierigkeiten zu der Personenfähigkeitsverteilung zu beurteilen, wurden die Verteilungen der WLEs und der Itemschwierigkeiten in Logit-Metrik gegenübergestellt. Im Rahmen vertiefender Analysen wurde die Messinvarianz des Instruments für Hochdeutsch sprechende bzw. dialektal geprägte Studierende geprüft (eindimensionales 1PL-Modell für zwei Gruppen in ConQuest). Was den Umgang mit fehlenden Werten anbelangt, so wurden nicht bearbeitete Items bei der Skalierung im Sinne nichtadministrierter Items betrachtet (also als fehlende Werte und nicht als inkorrekte Antwort). Den Maximum Likelihood-Schätzungen in Mplus (Muthén \& Muthén, 19982017) und ConQuest (Wu et al., 2007) lagen dabei jeweils sämtliche 271 Fälle zugrunde, wobei auch unter Not Missing At Random-Bedingungen (NMAR; Rubin, 1976) weitgehend unverzerrte Parameterschätzungen zu erwarten sind (Pohl, Gräfe \& Rose, 2014; Rose, von Davier \& Nagengast, 2016).

\section{Ergebnisse}

\section{Itemanalysen}

Für die 36 Items ergaben sich relative Lösungshäufigkeiten im Bereich von $7 \%$ bis $92 \%(M=43 \%)$. Die Anzahl fehlender Werte war insgesamt relativ gering $(\operatorname{Max}=33$, also $12 \%$ bei Item $22 ; M=3 \%$ ). Auf dieser Itembasis wurde ein eindimensionales 2PL-Modell berechnet, um die Diskriminationsparamter auf Einzelitemebene zu bestimmen. Für 22 der 36 Items lagen die Diskriminationsparameter oberhalb des Kriteriums von $\alpha=0.80$ (die Tabelle E1 wird im Elektronischen Supplement 2 zur Verfügung gestellt). Im Vergleich mit einem eindimensionalen 1PL-Modell (d.h. unter Annahme identischer Diskriminationsparameter) zeigte sich eine bessere Modellpassung des 2PL-Modells im Hinblick auf den AIC (1PL: AIC $=9$ 972.07; 2PL: AIC = 9 849.63). Für den BIC ergab sich eine Präferenz für das 1PL-Modell (1PL: BIC = 10 105.34; 2PL: $\mathrm{BIC}=10$ 108.98). Zusammenfassend fanden sich also Hinweise auf eine Verletzung der Annahme identischer Itemdiskriminationen. Entsprechend wurde eine Itemselektion anhand des Kriteriums $\alpha>0.80$ vorgenommen.

Anschließend wurden zur Überprüfung der verbleibenden 22 Items auf Modellkonformität im Hinblick auf ein einparametrisches logistisches Modell die gewichteten Abweichungsquadrate (WMNSQ) ermittelt. Alle Items rangierten im Wertebereich von $0.82 \leq$ WMNSQ $\leq 1.08$ (Tabelle 1).

Die entsprechenden kritischen $t$-Werte, die die Abweichung vom Erwartungswert von 1 schätzen, über- oder unterschritt keines der Items. Sie lagen im Bereich von $-1.2 \leq|t| \leq 1.9$ (Tabelle 1). Die anhand eines dreidimensionalen 1PL-Modells in ConQuest ermittelten Korrelationen zwischen den Dimensionen (die jeweils einen der operationsbezogenen Bereiche PI, PS, PM repräsentierten) lagen im Bereich .62 $\leq r \leq .76$, wobei sich für dieses Modell sowohl mit Blick auf den AIC (eindimensional: AIC $=5$ 910.17; dreidimensional: AIC $=5$ 854.13) als auch bezogen auf den BIC (eindimensional: BIC = 5 993.02; dreidimensional: $\mathrm{BIC}=5$ 954.99) eine bessere Passung zu den Daten ergab als für das eindimensionale Modell. Das Testlet-Modell (AIC $=5$ 891.62; BIC $=5$ 985.28) wurde ebenfalls anhand beider Fitindizes gegenüber dem eindimensionalen Modell präferiert. Bei exploratorischen 
Tabelle 1. Itemparameter aus einem 1PL-Modell auf Basis der verbleibenden 22 Items (geschätzt in ConQuest)

\begin{tabular}{cccccccccccc}
\hline Item & $\delta$ & \multicolumn{3}{c}{ WMNSQ } & \multicolumn{1}{c}{$t$} & Item & $\delta$ & & WMNSQ \\
\hline 2 & -0.21 & $(0.16)$ & 0.98 & $(0.89,1.11)$ & -0.4 & 20 & 1.97 & $(0.20)$ & 0.99 & $(0.79,1.21)$ & -0.1 \\
4 & -0.86 & $(0.16)$ & 1.05 & $(0.87,1.13)$ & 0.8 & 21 & 3.00 & $(0.26)$ & 0.82 & $(0.65,1.35)$ & -1.0 \\
6 & -0.08 & $(0.16)$ & 0.96 & $(0.89,1.11)$ & -0.7 & 22 & 1.83 & $(0.20)$ & 0.92 & $(0.80,1.20)$ & -0.8 \\
8 & 0.35 & $(0.16)$ & 1.07 & $(0.89,1.11)$ & 1.2 & 23 & -2.69 & $(0.23)$ & 1.04 & $(0.71,1.29)$ & 0.3 \\
9 & -0.83 & $(0.16)$ & 0.93 & $(0.87,1.13)$ & -1.1 & 26 & -0.65 & $(0.16)$ & 1.03 & $(0.88,1.12)$ & 0.4 \\
11 & -0.86 & $(0.16)$ & 0.97 & $(0.87,1.13)$ & -0.4 & 27 & 0.55 & $(0.16)$ & 1.04 & $(0.89,1.11)$ & 0.7 \\
13 & -1.85 & $(0.19)$ & 0.95 & $(0.81,1.19)$ & -0.5 & 29 & 0.63 & $(0.16)$ & 1.07 & $(0.88,1.12)$ & 1.1 \\
15 & 0.71 & $(0.16)$ & 1.06 & $(0.88,1.12)$ & 1.0 & 33 & 1.54 & $(0.18)$ & 1.00 & $(0.83,1.17)$ & 0.1 \\
16 & 1.50 & $(0.18)$ & 1.02 & $(0.84,1.16)$ & 0.2 & 34 & -0.42 & $(0.16)$ & 0.89 & $(0.88,1.12)$ & -1.9 \\
17 & 1.99 & $(0.20)$ & 1.07 & $(0.79,1.21)$ & 0.6 & 35 & 1.00 & $(0.17)$ & 1.05 & $(0.87,1.13)$ & 0.8 \\
19 & 3.19 & $(0.27)$ & 0.88 & $(0.61,1.39)$ & -0.6 & 36 & 0.94 & $(0.17)$ & 1.08 & $(0.87,1.13)$ & 1.2 \\
\hline
\end{tabular}

Anmerkungen: $N=271$; Itemschwierigkeit $\delta$ mit Standardfehler in Klammern; Weighted Mean Square-Kriterium (WMNSQ) mit 95\%-Konfidenzintervall in Klammern und $t$-Wert.

Faktorenanalysen mit drei Faktoren, die eine mögliche Dimensionierung anhand der Bereiche PI, PS und PM aufdecken sollte, zeigte sich ein Muster, das eher eine Dimensionierung auf Aufgabenebene als eine entsprechend der Bereiche PI, PS und PM nahelegte. So luden sämtliche fünf Items einer Aufgabe, bei der Minimalpaare erkannt werden sollten (PS), statistisch signifikant positiv auf den ersten Faktor $\left(.41 \leq \lambda_{\text {std }} \leq .81\right)$, auf den noch ein Item aus dem Bereich PI sowie zwei aus dem Bereich PS statistisch signifikant positive Ladungen aufwiesen. Der zweite Faktor repräsentierte im Wesentlichen einen Generalfaktor mit durchweg positiven $\left(.16 \leq \lambda_{\text {std }} \leq .97\right)$ und mit Ausnahme von insgesamt fünf Items - statistisch signifikanten Ladungen. Auf den dritten Faktor luden sämtliche vier Items einer Aufgabe (PS), bei der das Phonem an einer bestimmten Position eines Wortes erkannt werden sollte $\left(.53 \leq \lambda_{\text {std }} \leq .71\right)$, vier weitere Items aus dem Bereich PS sowie ein Item aus dem Bereich PI statistisch signifikant positiv. Die Ergebnisse sind allerdings vor dem Hintergrund der teilweise relativ großen Standardfehler der Ladungen $(0.03 \leq S E \leq 0.23)$ zu betrachten.

\section{Itemschwierigkeit}

Im Hinblick auf die Itemschwierigkeiten kann - bei einer Varianz der latenten Variable für die Personenparameter $\operatorname{von} \operatorname{Var}(\Theta)=1.36(S E=0.16)$ - auf einen Range von nahezu sechs Logits verwiesen werden, sodass die Variation der Phonembewusstheit in der Zielpopulation abgebildet werden kann (Abbildung 1).

Die mittlere Itemschwierigkeit betrug $M\left(\delta_{\mathrm{i}}\right)=0.49$ bei Fixierung der latenten Fähigkeitsvariable auf den Mittelwert Null. Zudem zeigte sich deskriptiv, dass die PI-Aufgaben im Mittel leichter für die untersuchte Stichprobe waren als die PS-Aufgaben und diese wiederum leichter als die PM-Aufgaben $\left(M_{\mathrm{PI}}\left(\delta_{\mathrm{i}}\right)=-1.10<M_{\mathrm{PS}}\left(\delta_{\mathrm{i}}\right)=0.09\right.$ $\left.<M_{\mathrm{PM}}\left(\delta_{\mathrm{i}}\right)=2.02\right)$. Bezüglich der Messgenauigkeit wurde im eindimensionalen Rasch-Modell eine Reliabilität von WLE PSR $=.80$ ermittelt. Die Präzision der Personenparameterschätzer variierte in der vorliegenden Stichprobe zwischen $S E=0.51$ bei WLE $=0.50$ und $S E=1.59$ bei WLE $=-4.40(M=0.58, M d n=0.54)$, wobei sich nur für $5 \%$ der Personen Standardfehler größer als $S E=0.78$ ergaben (dies entspricht hier etwa zwei Drittel einer Standardabweichung der Personenparameterverteilung).

Vertiefende Analysen ergaben Itemschwierigkeitsunterschiede zwischen Studierenden, die angaben, keinen Dialekt in alltäglichen Situationen zu sprechen $(n=45)$, und Studierenden, die zumindest gelegentlich Dialekt sprechen $(n=226$; Haupteffekt: 0.19 Logits niedrigere Itemschwierigkeiten für die Gruppe ohne Dialekt; $\chi^{2}(1)=$ $7.91 ; p=.005$ ). Auch für die Interaktionseffekte (Item $\mathrm{x}$ Gruppe) fanden sich statistisch signifikante Gruppenunterschiede $\left(\chi^{2}(22)=41.55 ; p=.007\right)$. Dabei ergaben sich für fünf der 22 Items Differenzen von mehr als 0.6 Logits (0.66 bis 0.98), die als "noteworthy for further investigation" (Pohl \& Carstensen, 2012, S. 12) eingestuft werden können. Für weitere vier Items fanden sich Differenzen im Bereich von 0.41 bis 0.48 Logits zwischen den Gruppen, die als "considerable but not sincerly" (Pohl \& Carstensen, 2012, S. 12) bewertet werden können.

\section{Diskussion}

Ziel der vorliegenden Studie war es, erstmalig für den deutschsprachigen Raum ein Instrument zur Erfassung der Phonembewusstheit nach schriftsprachlichem Input 


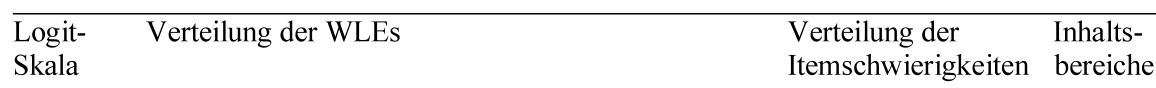

5

$\mathrm{X}$

4

$\mathrm{XX}$

$32 \quad \begin{array}{rr}19 & \text { PM } \\ 21 & P M\end{array}$

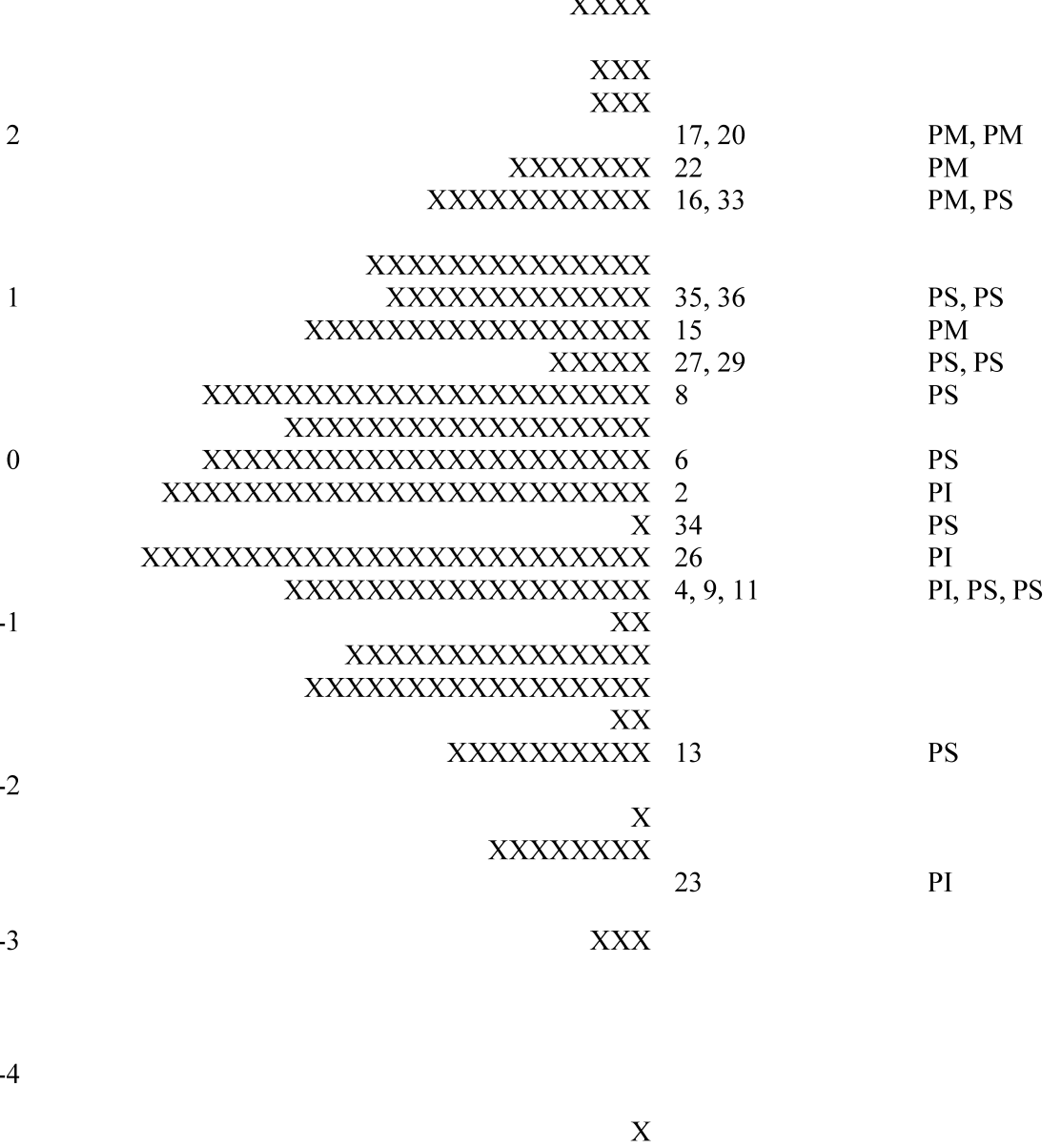

Anmerkungen: Jedes „X“ repräsentiert einen Fall; PM = Phoneme manipulieren; PS = Phoneme segmentieren; PI = Phoneme identifizieren.

Abbildung 1. Verteilung der Personenparameterschätzungen (WLE) und Itemschwierigkeiten bezogen auf die Itemauswahl (22 Items) auf Basis eines 1PL-Modells ( $N=271)$.

von angehenden Primarschullehrkräften zu erstellen und dessen psychometrische Güte zu überprüfen. Das entwickelte Verfahren namens TEPP fokussiert damit auf eine besonders wichtige Qualifikation von angehenden Primarschullehrkräften, um schriftsprachlichen Anfangsunterricht zu gestalten.

Die Aufgaben wurden basierend auf theoretischen Überlegungen zum Schriftspracherwerb und zum Kon- strukt der phonologischen Bewusstheit konzipiert. Vor dem Hintergrund empirischer Befunde zur Bedeutung der Phonembewusstheit für frühe Schriftsprachleistungen wurden Aufgaben speziell für diese linguistische Einheit konstruiert und dabei verschiedene Operationen (Identifizieren, Segmentieren, Manipulieren) berücksichtigt.

Die Itemanalysen zeigten, dass etwas mehr als die Hälfte der ursprünglich selektierten Items (22 von insge- 
samt 36 Items) auf Basis des Kriteriums eines Diskriminationsparameters von mindestens $\alpha=0.80$ beibehalten werden konnte. Dies ist ein relativ hoher Anteil, was auf eine sorgfältige Itementwicklung verweist. Inhaltlich decken die verbleibenden Items die angestrebte Breite des Konstrukts sehr gut ab, sodass die Inhaltsvalidität durch die Itemselektion unberührt bleibt.

In der vorliegenden Studie ergab sich eine mittlere Itemschwierigkeit von $M\left(\delta_{\mathrm{i}}\right)=0.49$, was bei einem auf Null fixierten Mittelwert und einer Streuung der Personenparameter von $S D=1.17$ bedeutet, dass der Test für Personengruppen mit einer um ca. eine halbe Standardabweichung höheren Leistung besser geeignet wäre. Insgesamt kann der Test somit für die Studierenden in der vorliegenden Stichprobe als tendenziell eher anspruchsvoll eingestuft werden bzw. - aus kriterialer Perspektive betrachtet - wiesen die Studierenden im Durchschnitt eher niedrige Kompetenzen auf. Bezogen auf eine Schwierigkeitsabstufung der unterschiedlichen Aufgabentypen sprechen die Ergebnisse insgesamt betrachtet erwartungsgemäß dafür, dass Aufgaben zum Identifizieren leichter sind als Aufgaben zum Segmentieren und diese wiederum leichter als Aufgaben zum Manipulieren.

Mit einer Reliabilität von .80 wäre die durchschnittliche Messgenauigkeit des Verfahrens bezogen auf die vorliegende Stichprobe für die Individualdiagnostik im Hinblick auf wichtige Entscheidungen (z.B. zur Erteilung von Zertifikaten) als unzureichend einzustufen, im Hinblick auf weniger weitreichende Entscheidungen (z. B. Empfehlungen zur Teilnahme an einer Fortbildungsmaßnahme) jedoch als hinreichend zu beurteilen (Evers, Sijtsma, Lucassen \& Meijer, 2010). Da es sich um ein IRTbasiertes Verfahren handelt, kann über die Reliabilität hinaus auch die Testinformation in Form des Standardfehlers der Punktschätzer zur Beurteilung herangezogen werden, um eine bessere Abschätzung der Messgenauigkeit des Tests abhängig vom (erwarteten) Leistungsniveau vornehmen zu können. In diesem Zusammenhang sei auch darauf hingewiesen, dass die Messgenauigkeit jeweils vor dem Hintergrund des geplanten Gesamtprozesses zu bewerten ist, wie z.B. der (erwarteten) Verteilung der Personenparameter in der Zielpopulation und der konkreten Fragestellung (Deutsches Institut für Normung e.V. [DIN], 2002; Hagemeister, Kersting \& Stemmler, 2012).

Interessant sind auch die Ergebnisse der Überprüfung der Messinvarianz des Instruments bezüglich dialektal geprägter versus Hochdeutsch sprechender Personen. Hier ergaben sich statistisch wie inhaltlich signifikante Unterschiede in den Itemschwierigkeiten zwischen den beiden Gruppen (differential item functioning, DIF; Zumbo, 1999), die im Einklang mit der theoretisch begründeten Annahme eines negativen Zusammenhangs zwischen Dialekt und phonologischer Bewusstheit stehen (Terry, 2014).

\section{Limitationen}

Trotz des akzeptablen Stichprobenumfangs ist bezüglich der Diskriminationsparameter die Schätzungenauigkeit zu berücksichtigen. Es ist durchaus möglich, dass auf Basis des Selektionskriteriums $(\alpha>0.80)$ in der vorliegenden Studie ausgeschlossene Items sich ggf. in einer weiteren (größeren) Stichprobe als akzeptabel erweisen könnten.

Auch die Frage der Dimensionalität von TEPP konnte im Rahmen dieser Studie trotz des relativ großen, aber für komplexe mehrdimensionale IRT-Modelle nicht optimalen Stichprobenumfangs nur eingeschränkt betrachtet werden. Die Ergebnisse legen nahe, dass TEPP in Teilen auch bereichsspezifische Facetten miterfasst, die allerdings nur bedingt eine Modellierung als eigenständige Konstrukte (d.h. als dreidimensionales Modell) andeuten, sondern - wie die Ergebnisse der exploratorischen Faktorenanalyse nahelegen - eher auf aufgabenspezifische Varianzanteile zurückgehen. Zudem wurde TEPP bislang nur für angehende Primarschullehrkräfte konzipiert und erprobt. Es ist aber davon auszugehen, dass es auch geeignet ist, um bei bereits tätigen Lehrkräften eingesetzt zu werden. Durch den Einsatz bei bereits tätigen Lehrkräften könnte überprüft werden, ob diese über eine höher ausgeprägte Phonembewusstheit verfügen als angehende Lehrkräfte. Internationale Studien liefern diesbezüglich uneinheitliche Ergebnisse (Bos, Mather, Dickson, Podhajski \& Chard, 2001; Washburn, Joshi \& Binks-Cantrell, 2011; Washburn et al., 2016). Des Weiteren wurde TEPP bislang nur bei angehenden Lehrkräften in BadenWürttemberg und Bayern (dort allerdings nur an einer Hochschule) eingesetzt. In anderen Bundesländern könnten sich basierend auf anderen Lehramtscurricula und dialektalen Unterschieden leicht andere Ergebnisse ergeben. Grundsätzlich sind jedoch ähnliche Ergebnisse zu erwarten.

In der vorliegenden Studie wurden hinsichtlich der Validität von TEPP Aspekte der Inhaltsvalidität (anhand von Expertenratings) und der faktoriellen Validität (konfirmatorische und exploratorische Faktorenanalysen) untersucht. Die Frage der prädiktiven Validität im Hinblick auf ein Kriterium wie beispielsweise die Vorhersage der Leistungen von Schülerinnen und Schülern bleibt ein Forschungsdesiderat.

\section{Fazit und Ausblick}

Die psychometrischen Gütekriterien von TEPP erwiesen sich bei ihrer Überprüfung als zufriedenstellend. Es kann 
somit davon ausgegangen werden, dass TEPP geeignet ist, um die Phonembewusstheit nach schriftsprachlichem Input von angehenden Lehrkräften akzeptabel zu erfassen.

Die explizite phonologische Bewusstheit von (angehenden) Lehrkräften kann sowohl in der Hochschulausbildung als auch in der beruflichen Weiterbildung erfolgreich verbessert und orthographische Interferenzeffekte reduziert werden (Brady et al., 2009; Cunningham, Etter, Platas, Wheeler \& Campbell, 2015; Purvis et al., 2016; Spear-Swerling \& Brucker, 2004). Eine verbesserte explizite phonologische Bewusstheit der Lehrkraft geht mit einer Verbesserung der Quantität und Qualität der Unterrichtsaktivitäten zur phonologischen Bewusstheit einher und führt darüber hinaus zu verbesserten (schriftsprachlichen) Leistungen bei den Kindern (Cunningham et al., 2015; Spear-Swerling \& Brucker, 2004). Der Einsatz von TEPP in Aus- und Weiterbildung kann demnach dazu dienen, wichtige Informationen über den Bedarf einer Adaption von (einzelnen) Lehrangeboten oder konzeptioneller Ausrichtungen von Lehrkräftefortbildungen zu generieren. Um TEPP noch besser für die Individualdiagnostik nutzbar zu machen, wird eine Ergänzung des aktuellen Itempools um weitere Aufgaben notwendig sein. Dabei wäre insbesondere eine Erweiterung um tendenziell leichte Items wünschenswert, da TEPP dann besser für Studienanfängerinnen und -anfänger geeignet wäre. Damit wäre eine regelmäßige und umfassende Evaluation der Phonembewusstheit über den gesamten Studienverlauf bei Grundschullehramtsstudierenden möglich. Durch den Einsatz von TEPP in zukünftigen Untersuchungen sollen zusätzliche empirische Ergebnisse generiert werden, die die hier präsentierten, ersten Erkenntnisse zur Güte des Instruments ergänzen und präzisieren.

\section{Elektronische Supplemente (ESM)}

Die elektronischen Supplemente sind mit der OnlineVersion dieses Artikels verfügbar unter https://doi.org/ 10.1026/0012-1924/a000272

ESM 1. Abgewandelte Beispielitems

ESM 2. Tabelle E1. Die Tabelle zeigt die Itemparameter aus einem eindimensionalen 2PL-Modell mit sämtlichen 36 Items.

\section{Literatur}

Akaike, H. (1974). A new look at the statistical model identification. IEEE Transactions on Automatic Control, 19, 716-722. https:// doi.org/10.1109/TAC.1974.1100705
Andrich, D. (1982). An index of person separation in latent trait theory, the traditional KR-20 index, and the Guttman scale response pattern. Educational Research and Perspectives, 9(1), $95-104$.

Aro, M. \& Björn, P. M. (2016). Preservice and inservice teachers' knowledge of language constructs in Finland. Annals of Dyslexia, 66, 111-126. https://doi.org/10.1007/s11881-015-0118-7

Ayala, R. J. de. (2009). The theory and practice of item response theory. New York, NY: The Guilford Press.

Berendes, K., Schnitzler, C. D., Willmes, K. \& Huber, W. (2010). Die Bedeutung von Phonembewusstheit und semantisch-lexikalischen Fähigkeiten für Schriftsprachleistungen in der Grundschule. Sprache - Stimme - Gehör, 34, 165-172. https://doi. org/10.1055/s-0029-1271722

Berendes, K. \& Weinert, S. (2016). Selecting appropriate phonological awareness indicators for the kindergarten cohort of the National Educational Panel Study: A theoretical and empirical approach. In H.-P. Blossfeld, J. von Maurice, M. Bayer \& J. Skopek (Eds.), Methodological issues of longitudinal surveys. The example of the National Educational Panel Study (pp. $401-$ 425). Wiesbaden: Springer VS. https://doi.org/10.1007/978-3658-11994-2_23

Berkemeier, A. \& Drinhaus, M. (2014). Förderung phonologischer Bewusstheit für den Schrift- und Orthographieerwerb in inklusiven Settings. In S. Trumpa, S. Seifried, E.-K. Franz \& T. Klauß (Hrsg.), Inklusive Bildung. Erkenntnisse und Konzepte aus Fachdidaktik und Sonderpädagogik (S. 308-320). Weinheim u. a.: Beltz Juventa.

Binks-Cantrell, E., Washburn, E. K., Joshi, R. M. \& Hougen, M. (2012). Peter Effect in the preparation of reading teachers. Scientific Studies of Reading, 16, 526-536. https://doi.org/10. 1080/10888438.2011.601434

Blatter, K. (2015). Familiale sprachbezogene Förderung und frühe (schrift-)sprachliche Kompetenzen - Zusammenhänge bei Kindern mit und ohne Migrationshintergrund. Inaugural-Dissertation in der Fakultät Humanwissenschaften der Otto-FriedrichUniversität Bamberg.

Bos, C., Mather, N., Dickson, S., Podhajski, B. \& Chard, D. (2001). Perceptions and knowledge of preservice and inservice educators about early reading instruction. Annals of Dyslexia, 51, 97 120. https://doi.org/10.1007/s11881-001-0007-0

Brady, S. A., Gillis, M., Smith, T., Lavalette, M., Liss-Bronstein, L., Lowe, E., et al. (2009). First grade teachers' knowledge of phonological awareness and code concepts. Examining gains from an intensive form of professional development and corresponding teacher attitudes. Reading and Writing, 22(4), 425-455. https://doi.org/10.1007/s11145-009-9166-x

Bus, A. G. \& ljzendoorn, M. H. van (1999). Phonological awareness and early reading: Meta-analysis of experimental training studies. Journal of Educational Psychology, 91, 403 - 414. https:// doi.org/10.1037/0022-0663.91.3.403

Cadinu, M., Maass, A., Rosabianca, A. \& Kiesner, J. (2005). Why do women underperform under stereotype threat? Evidence for the role of negative thinking. Psychological Science, 16, 572578. https://doi.org/10.1111/j.0956-7976.2005.01577.x

Caravolas, M., Volin, C. \& Hulme, C. (2005). Phoneme awareness is a key component of alphabetic literacy skills in consistent and inconsistent orthographies: Evidence from Czech and English children. Journal of Experimental Child Psychology, 92, 107 139. https://doi.org/10.1016/j.jecp.2005.04.003

Castles, A., Holmes, V. M., Neath, J. \& Kinoshita, S. (2003). How does orthographic knowledge influence performance on phonological awareness tasks? The Quarterly Journal of Experimental Psychology, 56, 445-467. https://doi.org/10.1080/0272 4980244000486 
Cheesman, E. A., McGuire, J. M., Shankweiler, D. P. \& Coyne, M. (2009). First-year teacher knowledge of phonemic awareness and its instruction. Teacher Education and Special Education, 32, 270 - 289. https://doi.org/10.1177/0888406409339685

Cunningham, A. E., Etter, K., Platas, L., Wheeler, S. \& Campbell, K. (2015). Professional development in emergent literacy: A design experiment of teacher study groups. Early Childhood Research Quarterly, 31, 62-77. https://doi.org/10.1016/j.ecresq.2014. 12.002

Diehl, K. (2017). Inklusion im Deutschunterricht. In B. Hartke (Hrsg.), Handlungsmöglichkeiten Schulische Inklusion. Das Rügener Modell kompakt (S. 57-98). Stuttgart: Kohlhammer.

Deutsches Institut für Normung e.V. [DIN]. (2002). DIN 33430: Anforderungen an Verfahren und deren Einsatz bei berufsbezogenen Eignungsbeurteilungen. Berlin: Beuth.

Driver, M. K., Pullen, P. C., Kennedy, M. J., Williams, M. C. \& Ely, E. (2014). Using instructional technology to improve preservice teachers' knowledge of phonological awareness. Teacher Education and Special Education, 1-21. https://doi.org/10.1177/ 0888406414537902

Ehri, L. C., Nunes, S. R., Willows, D. M., Schuster, B. V., YaghoubZadeh, Z. \& Shanahan, T. (2001). Phonemic awareness instruction helps children learn to read: Evidence from the National Reading Panel's meta-analysis. Reading Research Quarterly, 36, 250 - 283. https://doi.org/10.1598/RRQ.36.3.2

Einsiedler, F., Frank, A., Kirschhock, E.-A., Martschinke, S. \& Treinies, G. (2002). Der Einfluss verschiedener Unterrichtsmethoden auf die phonologische Bewusstheit sowie auf Lese- und Rechtschreibleistungen im 1. Schuljahr. Psychologie in Erziehung und Unterricht, 49, 194-209.

Evers, A., Sijtsma, K., Lucassen, W. \& Meijer, R. R. (2010). The Dutch review process for evaluating the quality of psychological tests: history, procedure, and results. International Journal of Testing, 10, 295 -317. https://doi.org/10.1080/15305058.2010. 518325

Fischer, M. Y. \& Pfost, M. (2015). Wie effektiv sind Maßnahmen zur Förderung der phonologischen Bewusstheit? Eine meta-analytische Untersuchung der Auswirkungen deutschsprachiger Trainingsprogramme auf den Schriftspracherwerb. Zeitschrift für Entwicklungspsychologie und Pädagogische Psychologie, 47, 35 - 51. https://doi.org/10.1026/0049-8637/a000121

Fricke, S., Stackhouse, J. \& Wells, B. (2007). Phonologische Bewusstheitsfähigkeiten deutschsprachiger Vorschulkinder - eine Pilotstudie. Forum Logopädie, 21(3), 14-19.

Gillon, G. (2004). Phonological awareness: From research to practice. New York, London: The Guilford Press.

Graefen, G. \& Liedke-Göbel, M. (2020). Germanistische Sprachwissenschaft. Deutsch als Erst-, Zweit- oder Fremdsprache (3., überarb. und erw. Aufl.). Tübingen: Narr Attempto.

Hagemeister, C., Kersting, M. \& Stemmler, G. (2012). Test reviewing in Germany. International Journal of Testing, 12, 185-194. https://doi.org/10.1080/15305058.2012.657922

Jeuk, S. (2017). Schriftspracherwerb im Anfangsunterricht in vielsprachigen Klassen. In B. Lütke, I. Petersen \& T. Tajmel (Hrsg.), Fachintegrierte Sprachbildung: Forschung, Theoriebildung und Konzepte für die Unterrichtspraxis (S. 269-285). De Gruyter Mouton.

Joshi, R. M., Washburn, E. K. \& Kahn-Horwitz, J. (2016). Introduction to the special issue on teacher knowledge from an international perspective. Annals of Dyslexia, 66, 1-6. https://doi. org/10.1007/s11881-015-0119-6

Klatte, M., Steinbrink, C., Bergström, K. \& Lachmann, T. (2017). Lautarium: Ein computerbasiertes Trainingsprogramm für Grundschulkinder mit Lese-Rechtschreibschwierigkeiten. Göttingen: Hogrefe.
Klicpera, C. \& Gasteiger-Klicpera, B. (1995). Psychologie der Leseund Schreibschwierigkeiten. Entwicklung, Ursachen, Förderung. Weinheim: Beltz, Psychologie-Verlag.

Klicpera, C., Schabmann, A., Gasteiger-Klicpera, B. \& Schmidt, B. (2017). Legasthenie - LRS. Modelle, Diagnose, Therapie und Förderung (5., überarb. und erw. Aufl.). München, Basel: Ernst Reinhardt Verlag.

Ministerium für Kultus, Jugend und Sport Baden-Württemberg (2016). Bildungsplan der Grundschule - Deutsch. VillingenSchwenningen: Neckar-Verlag GmbH.

Moats, L. C. (1994). The missing foundation in teacher education: Knowledge of the structure of spoken and written language. Annals of Dyslexia, 44, 81-102. https://doi.org/10.1007/ BF02648156

Moats, L. C. (1999). Teaching reading is rocket science: What expert teachers of reading should know and be able to do. Washington, DC: American Federation of Teachers.

Mußmann, J. (2012). Förderschwerpunkt Sprache im inklusiven Unterricht. Sonderpädagogik in Berlin, 1, 4-22.

Muter, V., Hulme, C., Snowling, M. \& Taylor, S. (1998). Segmentation, not rhyming, predicts early progress in learning to read. Journal of Experimental Child Psychology, 71(1), 3-27. https:// doi.org/10.1006/jecp.1998.2453

Muthén, L. K. \& Muthén, B. O. (1998-2017). Mplus user's guide (8th ed.). Los Angeles, CA: Muthén \& Muthén.

National Institute of Child Health and Human Development (NICHD). (2000). Report of the National Reading Panel. Teaching children to read: An evidence-based assessment of the scientific research literature on reading and its implications for reading instruction: Reports of the subgroups (NIH Publication No. $00-$ 4754). Washington, DC: U.S. Government Printing Office.

Pfost, M. (2015). Children's phonological awareness as a predictor of reading and spelling. Zeitschrift für Entwicklungspsychologie und Pädagogische Psychologie, 47, 123-138. https://doi.org/ 10.1026/0049-8637/a000141

Pfost, M., Blatter, K., Artelt, C., Stanat, P. \& Schneider, W. (2019). Effects of training phonological awareness on children's reading skills. Journal of Applied Developmental Psychology, 65, 101067. https://doi.org/10.1016/j.appdev.2019.101067

Pittman, R. T., Zhang, S., Binks-Cantrell, E., Hudson, A. \& Joshi, R. M. (2020). Teachers' knowledge about language constructs related to literacy skills and student achievement in low socioeconomic status schools. Dyslexia, 26, 200-219. https://doi. org/10.1002/dys.1628

Pohl, S. \& Carstensen, C. H. (2012). NEPS technical report - scaling the data of the competence tests (NEPS Working Paper No. 14). Bamberg: Otto-Friedrich-Universität.

Pohl, S., Gräfe, L. \& Rose, N. (2014). Dealing with omitted and notreached items in competence tests: Evaluating approaches accounting for missing responses in item response theory models. Educational and Psychological Measurement, 74, 423452. https://doi.org/10.1177/0013164413504926.

Polich, J., McCarthy, G., Wang, W. S. \& Donchin, E. (1983). When words collide: Orthographic and phonological interference during word processing. Biological Psychology, 16(3-4), 155-180. https://doi.org/10.1016/0301-0511(83)90022-4

Purvis, C. J., McNeill, B. C. \& Everatt, J. (2016). Enhancing the metalinguistic abilities of pre-service teachers via coursework targeting language structure knowledge. Annals of Dyslexia, 66(1), 55-70. https://doi.org/10.1007/s11881-015-0108-9.

Rose, N., Davier, M. von \& Nagengast, B. (2016). Modeling omitted and not-reached items in IRT models. Psychometrika, 1-25. https://doi.org/10.1007/s11336-016-9544-7.

Rost, J. (2004). Testtheorie und Testkonstruktion (2. Aufl.). Bern: Hans Huber. 
Rubin, D. B. (1976). Inference and missing data. Biometrika, 63 , 581 - 592. https://doi.org/10.1093/biomet/63.3.581

Schaefer, B., Stackhouse, J. \& Wells, B. (2016). Phonological awareness development in children with and without spoken language difficulties: A 12-month longitudinal study of German-speaking pre-school children. International Journal of Speech-Language Pathology, 19, 465-475. https://doi.org/10. 1080/17549507.2016.1221449

Schneider, W. (2017). Lesen und Schreiben lernen. Wie erobern Kinder die Schriftsprache? Berlin: Springer. https://doi.org/10. 1007/978-3-662-50319-5.

Schnitzler, C. D. (2008). Phonologische Bewusstheit und Schriftspracherwerb. Stuttgart: Georg Thieme. https://doi.org/10.1055/ b-002-21540

Schulte-Körne, G. (2001). Lese-Rechtschreibstörung und Sprachwahrnehmung. Psychometrische und neurophysiologische Untersuchungen zur Legasthenie (Pädagogische Psychologie und Entwicklungspsychologie, Bd. 14). Münster: Waxmann.

Schwarz, G. E. (1978). Estimating the dimension of a model. Annals of Statistics, 6, 461-464. https://doi.org/10.1214/aos/ 1176344136

Seidenberg, M. S. \& Tanenhaus, M. K. (1979). Orthographic effects on rhyme monitoring. Journal of Experimental Psychology, 5, 546-554. https://doi.org/10.1037/0278-7393.5.6.546

Seymour, P. H. K., \& Evans, H. M. (1994). Levels of phonological awareness and learning to read. Reading and Writing, 6, $221-$ 250. https://doi.org/10.1007/BF01027084

Smith, S. B., Simmons, D. C. \& Kameenui, E. J. (1998). Phonological awareness: Instructional and curricular basics and implications. In D. C. Simmons \& E. J. Kameenui (Eds.), What readning research tells us about children with diverse learning needs: Bases and basics (pp. 129 -140). Mahwah, NJ: Lawrence Erlbaum.

Sparks, R. L., Patton, J. \& Murdoch, A. (2014). Early reading success and its relationship to reading achievement and reading volume: replication of '10 years later'. Reading and Writing, 27, 189 - 211, https://doi.org/10.1007/s11145-013-9439-2

Spear-Swerling, L. \& Brucker, P. O. (2004). Preparing novice teachers to develop basic reading and spelling skills in children. Annals of Dyslexia, 54, 332 - 364. https://doi.org/10.1007/ s11881-004-0016-x

Spencer, E. J., Schuele, C. M., Guillot, K. M. \& Lee, M. W. (2008). Phonemic awareness skill of speech-language pathologists and other educators. Language, Speech, and Hearing Services in Schools, 39, 512-520. https://doi.org/10.1044/0161-1461 (2008/07-0080)

Stark, H. L., Snow, P. C., Eadie, P. A. \& Goldfeld, S. R. (2016). Language and reading instruction in early years' classrooms: the knowledge and self-rated ability of Australian teachers. Annals of Dyslexia, 66, 28 - 54. https://doi.org/10.1007/s11881015-0112-0

Statistisches Landesamt Baden-Württemberg (Hrsg.). (2018). Lehramtsabsolventinnen und -absolventen an den Hochschulen in Baden-Württemberg für die Lehrämter Grund- und Hauptschulen bzw. Grundschulen ab dem Prüfungsjahr 2000. Stuttgart: Statistisches Landesamt Baden-Württemberg.

Stock, C. \& Schneider, W. (2011). PHONIT: Ein Trainingsprogramm zur Verbesserung der phonologischen Bewusstheit und Rechtschreibleistung im Grundschulalter. Göttingen: Hogrefe.

Steinbrink, C. \& Lachmann, T. (2014). Lese-Rechtschreibstörung Grundlagen, Diagnostik, Intervention. Berlin: Springer Verlag.

Terry, N. P. (2014). Dialect variation and phonological knowledge: Phonological representations and metalinguistic awareness among beginning readers who speak nonmainstream American English. Applied Psycholinguistics, 35, 155-176. https://doi. org/10.1017/S0142716412000276
Tunmer, W. E. \& Hoover, W. A. (1992). Cognitive and linguistic factors in learning to read. In: P. B. Gough, L. E. Ehri, \& R. Treiman R. (Eds.) Reading Acquisition (pp. 175-214). Hillsdale NV: Lawrence Erlbaum Associates.

Wagner, R. K. \& Torgesen, J. K. (1987). The nature of phonological processing and its causal role in the acquisition of reading skills. Psychological Bulletin, 101, 192 - 212. https://doi.org/10. 1037/0033-2909.101.2.192

Wang, W.-C. \& Wilson, M. (2005). The Rasch testlet model. Applied Psychological Measurement, 29, 126 -149. https://doi.org/10. 1177/0146621604271053

Warm, T. A. (1989). Weighted likelihood estimation of ability in item response theory. Psychometrika, 54, 427 - 450. https://doi.org/ 10.1007/BF02294627

Washburn, E. K., Binks-Cantrell, E. S., Joshi, R. M., Martin-Chang, S. \& Arrow, A. (2016). Preservice teacher knowledge of basic language constructs in Canada, England, New Zealand, and the USA. Annals of Dyslexia, 66, 7-26. https://doi.org/10.1007/ s11881-015-0115-X

Washburn, E. K., Joshi, R. M. \& Binks-Cantrell, E. (2011). Teacher knowledge of basic language concepts and dyslexia. Dyslexia, 17, 165 -183. https://doi.org/10.1002/dys.426

Wu, M. L., Adams, R. J., Wilson, M. R. \& Haldane, S. A. (2007). ACER ConQuest version 2.0: Generalized item response modelling software. Camberwell: ACER Press.

Ziegler, J. C. \& Goswami, U. (2005). Reading acquisition, developmental dyslexia, and skilled reading across languages: a psycholinguistic grain size theory. Psychological Bulletin, 131, 3-29. https://doi.org/10.1037/0033-2909.131.1.3

Zumbo, B. D. (1999). A handbook on the theory and methods of differential item functioning (DIF): Logistic regression modeling as a unitary framework for binary and Likert-type (ordinal) item scores. Ottawa, Ontario, Canada: Directorate of Human Resources Research and Evaluation, National Defense Headquarters.

\section{Autorenschaften}

Karin Berendes und Wolfgang Wagner teilen sich die Erstautorenschaft.

Prof. Dr. habil. Karin Berendes, SRH Hochschule für Gesundheit, University of Applied Health Sciences, Campus Stuttgart, Ostendstr.77/3, 70188 Stuttgart, karin.berendes@srh.de

\section{Förderung}

Förderung im Rahmen des Programms „Projektförderung für NachwuchswissenschaftlerInnen" der Eberhard Karls Universität Tübingen.

Open Access-Veröffentlichung ermöglicht durch die Eberhard Karls Universität Tübingen

\section{ORCID}

Karin Berendes

(iD)ttps://orcid.org/0000-0002-2800-472X

Wolfgang Wagner

(iDhttps://orcid.org/0000-0001-9781-4630

\section{Dr. Wolfgang Wagner}

Hector-Institut für Empirische Bildungsforschung

Eberhard Karls Universität Tübingen

Europastraße 6

72072 Tübingen

wolfgang.wagner@uni-tuebingen.de 